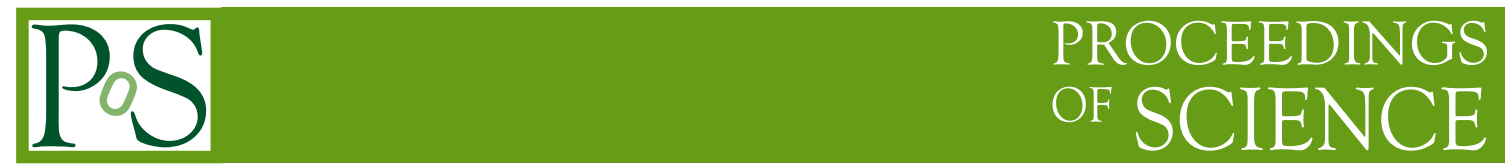

\title{
Studies of $\mathbf{W} / Z$ bosons at Tevatron
}

\author{
Sandra Leone*i \\ INFN Pisa, Italy \\ E-mail: sandra.leonedpi.infn.it
}

The Tevatron Collider provides $p \bar{p}$ collisions at a center of mass energy of $\sqrt{s}=1.96 \mathrm{TeV}$. Over the next few years the Tevatron is the only accelerator which can produce $W$ and $Z$ intermediate vector bosons. Measuring their properties is an important test of the standard model of elementary particles (SM). Significant deviations from SM predictions could indicate the presence of new physics. We present here some recent studies of $W$ and $Z$ properties made by the CDF and DØ Collaborations using data collected during Run II of the Tevatron.

International Europhysics Conference on High Energy Physics

July 21st - 27th 2005

Lisboa, Portugal

\footnotetext{
${ }^{*}$ Speaker.

$\dagger$ on behalf of the CDF and D $\varnothing$ Collaborations.
} 

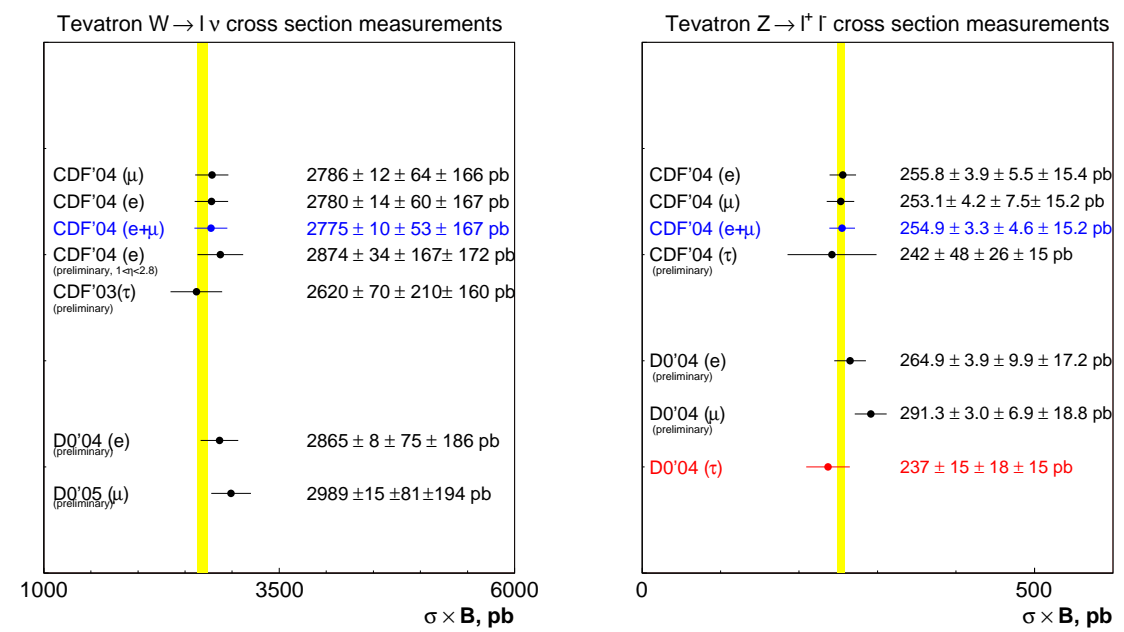

Figure 1: $\sigma(p \bar{p} \rightarrow W) \times B R\left(W \rightarrow \ell v_{\ell}\right)$ (left) and $\sigma(p \bar{p} \rightarrow Z) \times B R(Z \rightarrow \ell \ell)$ (right) measured at CDF and $\mathrm{D} \varnothing$. The uncertainties are listed in the following order: statistical, systematic and luminosity. The vertical band indicates the theoretical (NNLO) predictions.

\section{1. $W$ and $Z$ inclusive cross section measurements}

$W$ and $Z$ bosons are identified by their leptonic decays into electrons, muons and taus. Inclusive cross sections of both $W$ and $Z$ have been measured in all three lepton channels [1]. Figure 1 summarizes the $\mathrm{CDF}$ and $\mathrm{D} \emptyset$ cross section measurements. All measurements are in agreement with the NNLO calculations, represented by the vertical band [2]. The ratio $R$ of the cross section measurements for $W$ and $Z$ can be used to indirectly extract the total width of the $W$ boson. $R$ can be expressed as:

$$
R=\frac{\sigma(p \bar{p} \rightarrow W)}{\sigma(p \bar{p} \rightarrow Z)} \frac{\Gamma(W \rightarrow \ell v)}{\Gamma(Z \rightarrow \ell \ell)} \frac{\Gamma(Z)}{\Gamma(W)} .
$$

Inserting the SM predictions for the total cross sections and $\Gamma(W \rightarrow \ell v)$ and using the experimental $Z$ total and partial widths from LEP, one can extract $\Gamma(W)$. CDF measured the following values: $\Gamma(W)=2079 \pm 41 \mathrm{MeV}$, combining $e$ and $\mu$ channels and using $72 \mathrm{pb}^{-1}$ of data; and $\Gamma(W)=$ $2056 \pm 44 \mathrm{MeV}$ in the $\mu$ channel, using $194 \mathrm{pb}^{-1}$. These measurements are in agreement with both the PDG world average [3] and the SM prediction $(2091.1 \pm 2.5 \mathrm{MeV})$.

\section{Direct $W$ width measurement}

DØ measured directly the $W$ width in the electron channel using the transverse mass distribution, defined as:

$$
M_{T}=\sqrt{2 p_{T}^{\ell} p_{T}^{v}(1-\cos \Delta \phi)}
$$

where $p_{T}$ is the lepton transverse momentum and $\Delta \phi$ is the difference in azimuthal angle between the two leptons [4]. The width is determined by normalizing the signal and background $M_{T}$ distribution in the region of $50 \mathrm{GeV} / c^{2}<M_{T}<100 \mathrm{GeV} / c^{2}$ and fitting the predicted shape of the 

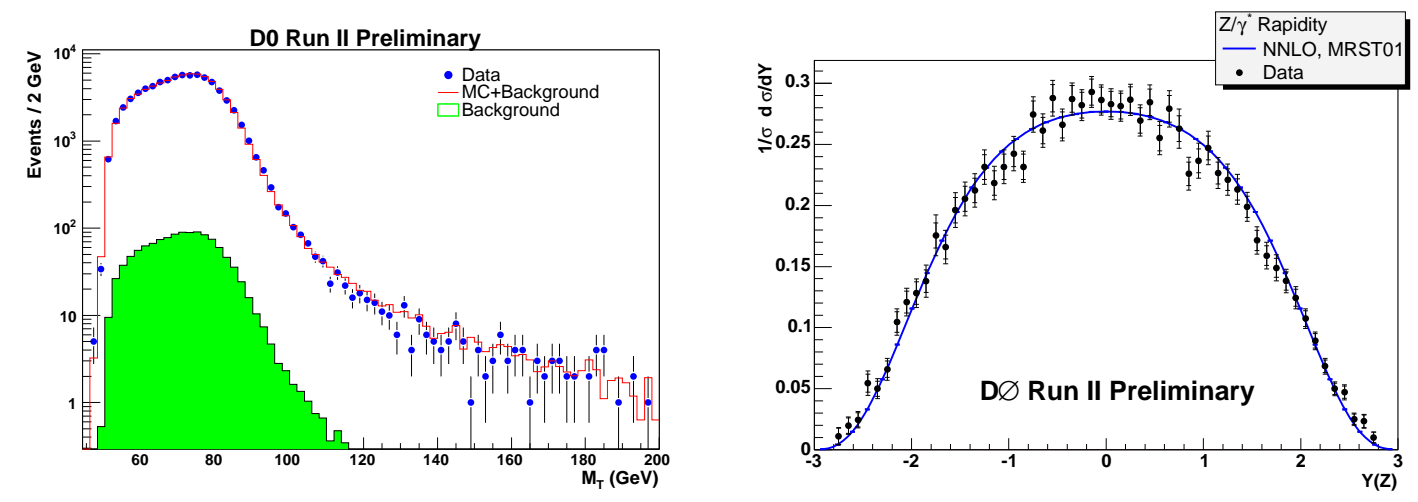

Figure 2: Left: Transverse mass distribution $M_{T}$ used by D $\varnothing$ for direct $W$ width determination. Right: $d \sigma / d y_{Z}$ measurement compared to NNLO prediction based on MRST01 PDF.

candidate events in the tail region $100 \mathrm{GeV} / c^{2}<M_{T}<200 \mathrm{GeV} / c^{2}$ which is most sensitive to the width. Figure 2 (left) shows the $M_{T}$ distribution. The measurement, obtained using $177 \mathrm{pb}^{-1}$ of data, yields: $\Gamma(W)=2011 \pm 93$ (stat) \pm 107 (syst) $\mathrm{MeV}$. The uncertainty is already smaller than that of the D $\emptyset$ Run I result.

\section{3. $Z$ rapidity distribution}

Measurement of the rapidity distribution of Drell-Yan pairs in the $Z$ boson mass region can provide a test of parton distribution functions (PDF) since the momentum fraction carried by the parton is directly related to the rapidity of the $Z$ boson $y_{Z}$. D $\varnothing$ measured the differential cross section $d \sigma / d y_{Z}$ of the Drell-Yan process in the dielectron's mass range $71 \mathrm{GeV} / c^{2}<M_{e e}<111$ $\mathrm{GeV} / c^{2}$, using $337 \mathrm{pb}^{-1}$ of data. Figure 2 (right) shows the measured $d \sigma / d y_{Z}$ distribution [5].

\section{4. $W$ charge asymmetry}

$W^{+}\left(W^{-}\right)$bosons are primarily produced through annihilation of valence $u(d)$ and $\bar{d}(\bar{u})$ quarks. Since $u$ quarks carry, on average, a higher fraction of the proton momentum than $d$ quarks, a $W^{+}\left(W^{-}\right)$tends to be boosted in the proton (anti-proton) direction. A measurement of the charge asymmetry in $W$ production, expressed as a function of the $W$ rapidity $y_{W}$, is sensitive to the ratio $u / d$ in PDF's. However, since the longitudinal component of the neutrino momentum is not measured, $y_{W}$ cannot be directly determined. Therefore we measure the electron charge asymmetry, defined as:

$$
A\left(\eta_{e}\right)=\frac{d \sigma\left(e^{+}\right) / d \eta_{e}-d \sigma\left(e^{-}\right) / d \eta_{e}}{d \sigma\left(e^{+}\right) / d \eta_{e}+d \sigma\left(e^{-}\right) / d \eta_{e}}
$$

where $\eta_{e}$ is the electron pseudorapidity. The observed asymmetry is a convolution of the $W$ production charge asymmetry and the $V-A$ asymmetry of the $W$ decay. CDF has made this measurement on a data sample of $170 \mathrm{pb}^{-1}$. Figure 3 (left) shows the measured asymmetry corrected for the effect of charge misidentification and background contributions [6]. 

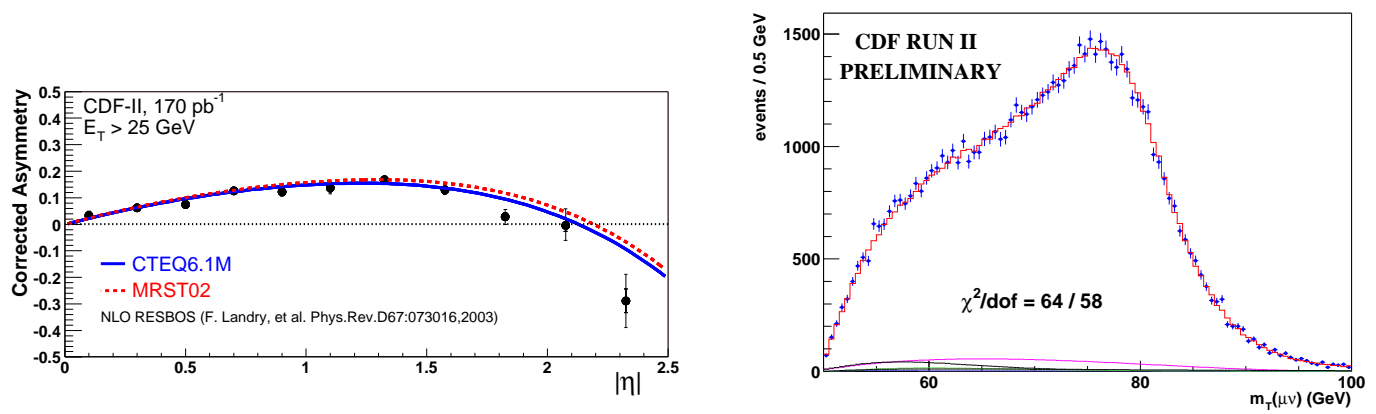

Figure 3: Left: $W$ charge asymmetry as a function of the electron $\eta$. The predictions using different PDF's are shown. Right: $M_{T}$ spectrum for $W \rightarrow \mu v$ candidate events at CDF. The histogram represents the simulation with background contribution added. The region between $60-90 \mathrm{GeV} / c^{2}$ is used to fit the $M_{W}$.

\section{5. $W$ Mass}

The $W$ mass $\left(M_{W}\right)$ measurement, together with the top quark mass, constrains, in the framework of the SM, the mass of the unobserved Higgs boson. $M_{W}$ is measured from a maximum likelihood fit to the transverse mass spectrum in the $e v$ and $\mu v$ channels. There are two main components leading to a precise $M_{W}$ measurement: calibration of the charged lepton momenta and of the energy of hadrons recoiling off the $W$ boson. CDF has estimated the $W$ mass uncertainty in an analysis using $200 \mathrm{pb}^{-1}$ of Run II data. Figure 3 (right) shows the $M_{T}$ distribution (muon channel) compared to the simulation and the expected background contributions. The current overall uncertainty is $76 \mathrm{MeV} / \mathrm{c}^{2}$, already smaller than CDF Run I result [7]. The $M_{W}$ fit results are currently blinded with a constant offset which will be removed when further cross checks are completed.

\section{Conclusions}

The Run II of the Tevatron is well underway. Both CDF and DØ are producing interesting results in the electroweak sector. D $\emptyset$ has made the first Run II direct measurement of the $W$ width. CDF has determined the uncertainty on the $W$ mass with the first $200 \mathrm{pb}^{-1}$ of Run II data to be 76 $\mathrm{MeV} / \mathrm{c}^{2}$. Run II will provide the world's highest precision measurement of the $W$ boson mass (30 $\mathrm{MeV} / \mathrm{c}^{2}$ for $2 \mathrm{fb}^{-1}$ of analyzed data).

\section{References}

[1] D. Acosta et al., Phys. Rev. Lett. 94, 091803 (2005) [hep-ex/0 406078 ].

[2] C. Anastasiou et al., Phys. Rev. D 69, 094008 (2004) [hep-ph/ 0312266$].$

[3] S. Eidelman et al., Physics Letters B 592, 1 (2004).

[4] DØ note 4563-CONF (2004).

[5] DØ note http://www-d0.fnal.gov/Run2Physics/WWW/results/prelim/EW/E12/E12.pdf.

[6] D. Acosta et al., Phys. Rev. D 71, 051104 (2005) [hep-ex/ 0501023 ].

[7] T. Affolder et al., Phys. Rev. D 64, 052001 (2001) [hep-ex/0007044]. 